\title{
MANAGING CORRELATED STOCK EXTERNALITIES : WATER TAXES WITH A PINCH OF SALT
}

\author{
SOPHIE LEGRAS \\ Université de Montpellier 1, INRA LAMETA \\ ANU, Crawford School of Economics and Government
}

\begin{abstract}
In this paper we address the design of dynamic taxation schemes in the presence of multiple interacting stocks. After addressing a general model, we focus the analysis on the context of irrigation-induced salinity. We study various taxes, based either on a collective performance measure (groundwater stock) or on an individual performance measure (irrigation water, rootzone salt stock) or a mix of both. We show the interest for mixed tax schemes to induce agents to follow the optimal input use and stock accumulation paths. Keywords : Irrigation-induced salinity, taxation schemes, multiple state variables, differential game.
\end{abstract}

\section{INTRODUCTION}

A number of the most stringent environmental issues are dynamic by nature as they involve the accumulation of pollution or the depletion of resources. Examples include acid rains, climate change, ozone depletion, groundwater depletion or biodiversity loss. Most economic analyses address the case of a unique stock. A stream of literature has developed around the issue of designing efficient policy instruments in such inherently dynamic contexts. Ko et al. [14] adapt the standard Pigovian tax to a dynamic setting and compare flexible, but difficult to implement, taxes with inflexible, time independent, ones. Karp and Livernois [13] analyse a linear Markov perfect tax rule to induce a monopoly to exploit a non-renewable resource optimally. Hence the resource stock is introduced as a basis for taxation. Benchekroun and van Long [1] adapt this tax to the context of a polluting oligopoly. Both studies show how mixed tax schemes, based on individual and collective performance measures, are efficient in managing a dynamic externalities.

Fewer papers recognize that in some settings it is more appropriate to consider multiple interrelated stocks. This is quite straightforward in the case of predator-prey interactions [17]. Forest ecosystems are also illustrative of complex interactions between multiple

Date: May 10, 2007.

The author would like to thank Mabel Tidball, Robert Lifran and participants at the Workshop on Dynamic Economics at the School of Economics \& Finance, Victoria University of Wellington, for helpful comments. All remaining errors are the sole responsibility of the author. 
species. Crépin [6] and Borgland et al. [2] study the case of boreal forests by describing the interactions arising between various stocks, moose, caduceus and conifers in the case of [6]. Bond and Farzin [3] develop a multiple nutrient stocks model to highlight the differentiatied impacts of various decisions - fertilizer input, tillage - on the soil treated as a portfolio of nitrogen pools. Farzin and Tahvonen [9] and more recently, Caplan and Silva [5] or Yang [22], have pointed out the need to model climate change more accurately by describing multiple interacting stocks. Caplan and Silva [5] analyze pollution rights markets to manage carbon, a global pollutant, and smog, a local pollutant, emitted jointly by a set of firms. They show how a system combining a global market and regional markets is Pareto efficient. Yang [22] focuses on negatively correlated externalities in the same context, stemming from the observation that primary energy consumption generates a global externality, global warming, and a local externality, SO2 emission, that alleviates global warming and in this sense is at the same time harmful to local air quality and beneficial at the global level through its cooling effect. Yang [22] shows how the negative relationship between the global and the local externalities impacts on the design of policy instruments, as it raises the need for a subsidy for local externalities when accounting for the global issue.

Our aim in this paper is to address the design of mixed taxation schemes, in the vein of Benchekroun and van Long [1], when a global stock and multiple individual stocks interact. In this regard, the questions we pose in this paper is whether an efficient taxation scheme to manage interacting stocks should be based on individual performance (input use, individual stock), collective performance (global stock) or a mix of both.

We base our study on the analysis of a particularly stringent environmental issue that affects irrigation districts of most semiarid areas of the world, namely irrigation-induced salinity. In these regions, the development of irrigation has induced various environmental damage, known as the twin menace of irrigation, salinization and waterlogging. These phenomena, which may occur naturally, are worsened by inappropriate irrigation practices. The extent of the problem is worldwide and increasing. The World Bank reports that 24 per cent of irrigated areas are severely affected and that around 10 millions hectares of arable land are put out of production each year [19]. The extent of this environmental issue is such that it questions the sustainability of irrigation itself. Until now, management strategies that have been developed include the building of ad hoc infrastructures (large-scale drainage projects), farm-level initiatives (information and incitations to adopt better irrigation practices) or community-based voluntary programs, which have not proven efficient in the implementation phase. In order to address the incentive gaps existing in the context of irrigation, between the scarcity value of water and its current management value [18] and between the individual and social valuation of the aquifer as a stock of water, responsible for salinity and waterlogging [20], we analyze various types of water taxes and extend Legras and Lifran's [15] analysis to a two-stocks issue. In this context, individual performance is associated to the level of irrigation water, 
and the individual stock of rootzone salts, and collective performance corresponds to the accumulation of groundwater.

The contributions of this paper are twofold. First, we extend the analysis of dynamic taxation scheme design to the case of two interacting stocks. Second, we provide a detailed model of irrigation-induced salinity, that captures the interactions between water and salt.

The paper is organized as follows. In section 2, we analyze a general model of interrelated stocks, and highlight some properties of various tax schemes. In section 3 , we derive the social optimal and individual paths of irrigation water use and rootzone and groundwater stocks' accumulation. In section 4 we derive the optimal tax parameters. Finally, we collect some concluding remarkts in Section 5.

\section{A GENERAL MODEL OF INTERRELATED STOCKS}

Suppose the existence of a benevolent policymaker, whose program is to maximise total benefits, accounting for the existence of two sources of damage, a global stock $X$ and multiple local stocks, $Q_{i}$, for $i \in\{1 . . n\}$. Let $B_{i}\left(u_{i}, Q_{i}, X\right)$ be agent $i^{\prime}$ s benefit function, $u_{i}$ his control variable, with $\partial B_{i} / \partial Q_{i}<0$ and $\partial B_{i} / \partial X<0^{1}$. Social damage is denoted by $D(X)$ and $r$ is the discount rate. The socially optimal maximisation program is :

$$
\max _{\left\{u_{i}\right\} \geq 0} \int_{0}^{\infty}\left(\sum_{i} B_{i}\left(u_{i}, Q_{i}, X\right)-D(X)\right) e^{-r t} . d t \text {, subject to : }
$$

$$
\begin{aligned}
& \dot{X}=F\left(\sum_{i} u_{i}, X\right), \\
& \dot{Q}_{i}=G_{i}\left(u_{i}, Q_{i}, X\right), \forall i .
\end{aligned}
$$

This model is based on the one developped by Yang [22]. The main difference is that it is the collective stock that impacts on the individual ones rather than the opposite. Hence in the present framework $\partial G_{i} / \partial X \neq 0$ and $\partial F / \partial Q_{i}=0$, illustrating a type of feedback loop between the collective stock, an aggregation of individual decisions, and the individual stocks.

The Hamiltonian writes as follows :

$$
H^{s o}=\sum_{i=1}^{n} B_{i}\left(u_{i}, Q_{i}, X\right)-D(X)+\lambda^{s o} F\left(\sum_{i} u_{i}, X\right)+\sum_{i=1}^{n} \mu_{i}^{s o} G_{i}\left(u_{i}, Q_{i}, X\right),
$$

\footnotetext{
${ }^{1}$ To come back to the standard case where the agents are not affected by the stocks, simply set $\partial B_{i} / \partial Q_{i}=$ $\partial B_{i} / \partial X=0$.
} 
where $\lambda^{s o}$ and $\mu_{i}^{s o}$ are the costate variables associated with the collective and individual stocks, so that the first order conditions are given by equations (3)-(5) :

$$
\begin{aligned}
& \frac{\partial H}{\partial u_{i}}=\frac{\partial B_{i}}{\partial u_{i}}+\lambda^{s o} \frac{\partial F}{\partial u_{i}}+\mu_{i}^{s o} \frac{\partial G_{i}}{\partial u_{i}}=0, \\
& -\frac{\partial H}{\partial X}=\dot{\lambda}^{s o}-r \lambda^{s o}=-\left[\sum_{i=1}^{n} \frac{\partial B_{i}}{\partial X}-D^{\prime}(X)+\lambda^{s o} \frac{\partial F}{\partial X}+\sum_{i=1}^{n} \mu_{i}^{s o} \frac{\partial G_{i}}{\partial X}\right], \\
& -\frac{\partial H}{\partial Q_{i}}=\dot{\mu}_{i}{ }^{s o}-r \mu_{i}^{s o}=-\left[\frac{\partial B_{i}}{\partial Q_{i}}+\mu_{i}^{s o} \frac{\partial G}{\partial Q_{i}}\right], \forall i .
\end{aligned}
$$

Equation (3) can be rearranged to obtain expressions of the co-state variables :

$$
\lambda^{s o}=\frac{-\frac{\partial B_{i}}{\partial u_{i}}-\mu_{i}^{s o} \frac{\partial G_{i}}{\partial u_{i}}}{\frac{\partial F}{\partial u_{i}}} \text { and } \mu_{i}^{s o}=\frac{-\frac{\partial B_{i}}{\partial u_{i}}-\lambda^{s o} \frac{\partial F}{\partial u_{i}}}{\frac{\partial G_{i}}{\partial u_{i}}} .
$$

Then equations (4) and (5) become ${ }^{2}$ :

$$
\begin{aligned}
& {\left[\frac{\dot{\lambda}^{s o}}{\lambda^{s o}}-\left(r-\frac{\partial F}{\partial X}\right)\right] \frac{1}{\frac{\partial F}{\partial u_{i}}}=\frac{\sum_{i} \frac{\partial B_{i}}{\partial X}-D^{\prime}(X)+\sum_{i} \mu_{i} \frac{\partial G_{i}}{\partial X}}{\frac{\partial B_{i}}{\partial u_{i}}+\mu_{i}^{s o} \frac{\partial G_{i}}{\partial u_{i}}},} \\
& {\left[\frac{\dot{\mu}_{i}^{s o}}{\mu_{i}^{s o}}-\left(r-\frac{\partial G_{i}}{\partial Q_{i}}\right)\right] \frac{1}{\frac{\partial G_{i}}{\partial u_{i}}}=\frac{\frac{\partial B_{i}}{\partial Q_{i}}}{\frac{\partial B_{i}}{\partial u_{i}}+\lambda^{s o} \frac{\partial F}{\partial u_{i}}} .}
\end{aligned}
$$

The RHS of equations (6) and (7) present the ratio between the marginal desutilities from the stocks and the marginal utilities from irrigating, in other words a marginal rate of substitution. The numerator of the RHS of equation (6), for instance, includes the sum of direct individual damage from the global stock, $\sum_{i} \frac{\partial B_{i}}{\partial X}<0$, the social damage from the global stock, $-D^{\prime}(X)<0$, and the indirect impact of the global stock on the local stocks, $\sum_{i} \mu_{i}^{s o} \frac{\partial G}{\partial X}$. The latter illustrates the interaction between the stocks, that can be positive or negative. Hence if the stocks are positively correlated, $\frac{\partial G_{i}}{\partial X}>0$; as $\mu_{i}^{s o}<0$ the indirect impact of the global stock through the local stock acts as a damage. However, if the stocks are negatively correlated, this indirect impact is a benefit, as increasing the global stock tends to decrease the local one. Note that in equation (7) there is no interaction term, as it is the global stock that impacts on the local ones. The denominators of the RHS present the marginal utilities from using irrigation water, which is the marginal production plus a negative term accounting for the impact of increasing input use on the other stock.

The LHS of equations (6) and (7) are constituted of a dynamic term and of the marginal individual contribution to the (individual or collective) stock pollution. The dynamic term comprises the time path of the costate variables, for instance $\dot{\mu}_{i}{ }^{s o} / \mu_{i}^{s o}$ and an environmental discount rate, $r-\partial G_{i} / \partial Q_{i}$, where $\partial G_{i} / \partial Q_{i}$ is the natural stock dissipation rate.

Now, consider the case of individual agents following open-loop strategies. The individual maximisation program is :

\footnotetext{
${ }^{2}$ Assuming strict negativity of the co-state variables.
} 


$$
\max _{u_{i} \geq 0} \int_{0}^{\infty} B_{i}\left(u_{i}, Q_{i}, X\right) e^{-r t} . d t \text {, subject to equations (1) and (2). }
$$

The conditions driving the accumulation paths are :

$$
\begin{aligned}
& {\left[\frac{\dot{\lambda}^{o l}}{\lambda^{o l}}-\left(r-\frac{\partial F}{\partial X}\right)\right] \frac{1}{\frac{\partial F}{\partial u_{i}}}=\frac{\frac{\partial B_{i}}{\partial X}+\mu_{i}^{o l} \frac{\partial G_{i}}{\partial X}}{\frac{\partial B_{i}}{\partial u_{i}}+\mu_{i}^{o l} \frac{\partial G_{i}}{\partial u_{i}}},} \\
& {\left[\frac{\dot{\mu}_{i}^{o l}}{\mu_{i}^{o l}}-\left(r-\frac{\partial G_{i}}{\partial Q_{i}}\right)\right] \frac{1}{\frac{\partial G_{i}}{\partial u_{i}}}=\frac{\frac{\partial B_{i}}{\partial Q_{i}}}{\frac{\partial B_{i}}{\partial u_{i}}+\lambda^{o l} \frac{\partial F}{\partial u_{i}}} .}
\end{aligned}
$$

Comparing with the social optimum case, equations (8) and (9) highlight multiple sources of inefficiency. First, from equation (8), it appears that the social damage is not accounted for. Furthermore, individual agents account only for their individual damage, without taking into consideration the impact of a rising of the stock on the other agents. Also, in equation (8), $\mu_{i}$ isn't valued at the same level as in equation (6). Consequently, none of the stocks is accumulated optimally.

Suppose that the policymaker wishes to induce the agents to act optimally by implementing a taxation scheme. He consider various taxation bases : individual input use, individual stock, global stock:

$$
\tau\left(u_{i}, Q_{i}, X\right)=u_{i}\left(\tau_{1}+\tau_{2} X\right)+\tau_{3} X+\tau_{4} Q_{i} .
$$

Then individual decisions are modified in such a way as to lead to the following conditions on stock accumulation :

$$
\begin{aligned}
& {\left[\frac{\dot{\lambda}^{\tau}}{\lambda^{\tau}}-\left(r-\frac{\partial F}{\partial X}\right)\right] \frac{1}{\frac{\partial F}{\partial u_{i}}}=\frac{\frac{\partial B_{i}}{\partial X}+\mu_{i}^{\tau} \frac{\partial G_{i}}{\partial X}-\left(\tau_{2} u_{i}+\tau_{3}\right)}{\frac{\partial B_{i}}{\partial u_{i}}+\mu_{i}^{\tau} \frac{\partial G_{i}}{\partial u_{i}}-\left(\tau_{1}+\tau_{2} X\right)},} \\
& {\left[\frac{\dot{\mu}_{i}^{\tau}}{\mu_{i}^{\tau}}-\left(r-\frac{\partial G_{i}}{\partial Q_{i}}\right)\right] \frac{1}{\frac{\partial G_{i}}{\partial u_{i}}}=\frac{\frac{\partial B_{i}}{\partial Q_{i}}-\tau_{4}}{\frac{\partial B_{i}}{\partial u_{i}}+\lambda^{\tau} \frac{\partial F}{\partial u_{i}}-\left(\tau_{1}+\tau_{2} X\right)} .}
\end{aligned}
$$

Tax parameters $\left(\tau_{1}, \tau_{2}, \tau_{3}, \tau_{4}\right)$ don't have the same impact on the optimal paths. $\tau_{1}$ only appears in the expressions of marginal benefits from input use, $\tau_{3}$ in the expression of marginal disutility from global stock accumulation and $\tau_{4}$ in the expression of marginal disutility from local stock accumulation. $\tau_{2}$ plays a particular role as it is the interaction term, attached to both input use and the global stock.

Finally, consider the case of individual agents following feedback strategies. Assuming the existence of a MPNE, the Hamiltonian is :

$$
H^{f b}=B_{i}\left(u_{i}, Q_{i}, X\right)+\lambda^{f b} F\left(u_{i},\left\{u_{j}\left(Q_{j}, X\right)\right\}_{j \neq i}, X\right)+\mu_{i}^{f b} G_{i}\left(u_{i}, Q_{i}, X\right) .
$$


Then the same type of conditions are derived as in the previous cases describing the costate variables time paths :

$$
\begin{aligned}
& {\left[\frac{\dot{\lambda}^{f b}}{\lambda^{f b}}-\left(r-\frac{\partial F}{\partial X}+\sum_{j \neq i} \frac{\partial F}{\partial u_{j}} \frac{\partial u_{j}}{\partial X}\right)\right] \frac{1}{\frac{\partial F}{\partial u_{i}}}=\frac{\frac{\partial B_{i}}{\partial X}+\mu_{i}^{f b} \frac{\partial G_{i}}{\partial X}}{\frac{\partial B_{i}}{\partial u_{i}}+\mu_{i}^{f b} \frac{\partial G_{i}}{\partial u_{i}}}} \\
& {\left[\frac{\mu_{i}^{f b}}{\mu_{i}^{f b}}-\left(r+\frac{\partial G_{i}}{\partial Q_{i}}\right)\right] \frac{1}{\frac{\partial G_{i}}{\partial u_{i}}}=\frac{\frac{\partial B_{i}}{\partial Q_{i}}}{\frac{\partial B_{i}}{\partial u_{i}}+\lambda^{f b} \frac{\partial F}{\partial u_{i}}} .}
\end{aligned}
$$

In the LHS of equation (12), the summation term illustrates the difference between openloop and feedback decisions; indeed in the latter case, agents account for the impact of their decisions on the others' decisions. The direction of this impact depends on the sign of two terms. First, $\partial F / \partial u_{j}>0$ in the case of stock accumulation. Second, the sign of $\partial u_{j} / \partial X$ depends on the complement - substitute nature of agents' strategies [4]. If they are strategic complements, $\partial u_{j} / \partial X>0$, then the resulting environmental discount rate perceived by $i$ is increased. Consequently, agents $j$ increase their contribution faced to an increase of groundwater, and $i$ is induced to reduce his contribution to the stock. When $\partial u_{j} / \partial X<0$, agents $j$ lower their contributions as groundwater accumulates : $i$ is induced to contribute more to the accumulation of the stock.

Note that the consideration of individual local stocks simplifies the setting; indeed if the local stocks were managed by numerous agents, another layer of strategic interaction would exist.

Applying a tax $\tau\left(u_{i}, Q_{i}, X\right)=u_{i}\left(\tau_{1}+\tau_{2} X\right)+\tau_{3} X+\tau_{4} Q_{i}$ has the same effect as in the open-loop case. However the derivation of the optimal tax parameters needs to account for the altered 'environmental discount rate'.

The remainder of this paper presents irrigation-induced salinity as an illustrative example of a setting combining interacting individual and collective stock pollution.

\section{STOCK ACCUMULATION AND IRRIGATION DECISIONS IN THE CONTEXT OF IRRIGATION-INDUCED SALINITY}

In this section we illustrate the case of positively correlated externalities in the context of irrigation-induced salinity. We focus on the case of one catchment, understood as the recharge area of a specific aquifer. Above this aquifer, $n$ agents divert water from a river to undertake irrigation. In doing so, they generate a certain amount of water percolating down to the aquifer, and a certain amount of water available for uptake by plants in the rootzone. Each water reservoir (river, root-zone, watertable) is also characterized by its salt content. To highlight the main mechanisms associated with irrigation-induced 
salinity, we focus on the collective stock of groundwater and on the individual root-zone salt stocks ${ }^{3}$.

\subsection{The model.}

3.1.1. Watertable dynamics. We keep a simple description of watertable dynamics that accounts for the main mechanisms at stake. This allows us to focus on the impact of agents' decision on the state of the watertable :

$$
\dot{X}=\sum_{i=1}^{n} P_{i}-(\delta+\gamma) X,
$$

where $X$ is the stock of groundwater, $P_{i}$ is the water percolating from farm $i, \delta$ is a discharge fraction and $\gamma$ is a capillary rise parameter ${ }^{4}$.

3.1.2. Rootzone salts dynamics. We associate each agent with an individual salt stock.

The first source of salinity in the context of irrigation is poor quality irrigation water. Denoting $S^{W}$ the salt concentration of irrigation water, the first inflow of salt is $S^{W} u_{i}$. The second source of salts for the rootzone is the capillary rise from the watertable. Groundwater is an important tank of salts, bringing salts to the rootzone by capillary rise which constitutes the second source of salts for the rootzone. Denoting $S^{G}$ the salt concentration in groundwater, $\gamma_{i}$ an individual capillary rise factor, this inflow is : $\gamma_{i} S^{G} X$.

Percolation represents an outflow of salts from the rootzone, to the watertable. Denoting $S^{R}$ the salt concentration in the rootzone, then the amount of percolated salts is $S^{R} P_{i}$, where $P_{i}$ is the quantity of water percolating. Water remaining the the rootzone, available for uptake by plants, and water percolating are function of the quantity of applied water and the efficiency of the technology in use, $\beta: R_{i}=\beta u_{i}$ and $P_{i}=(1-\beta) u_{i}$.

Taking these mechanisms into account, an equation describing the dynamics of the quantity of salt in the root zone at location $i$ can be formulated as follows, with $Q_{i}$, the quantity of salts for farm $i$, and $S^{R}=Q_{i} / R_{i}$ :

$$
\dot{Q}_{i}=S^{W} u_{i}+\gamma S^{G} X-\frac{1-\beta}{\beta} Q_{i}
$$

The quantity of groundwater $X$, is a variable common to all the farmers, so is the concentration of salt $S^{G}$. Furthermore, $S^{W}$ and $S^{G}$ are treated as exogenous variables. However, water remaining in the rootzone and percolating water are individual variables, as they depend on individual choices, regarding the quantity of water applied and the irrigation technology.

3.1.3. Different types of damages. Irrigation-induced salinity is a complex environmental issue, as it combines problems associated with both the quantity and the quality of water, at two levels, the surface system and the underground system. In this paper, we consider

\footnotetext{
${ }^{3}$ Hence we don't account for the interactions that exist between the river and the aquifer explicitely.

$4_{0} \leq \delta \leq 1$ and $0 \leq \gamma \leq 1$.
} 
three main sources of damage : soil salinity and waterlogging are treated as individual damage, affecting directly the agents' production function, while the discharge of salty water is treated as a social damage, which individual irrigators do not account for.

Rootzone salinity. The primary effect of a saline water in the rootzone is the inability of the plant to compete for water with ions in the soil solution, a phenomenon known as physiological drought. The higher the salinity the less water available to plants. This has negative consequences in terms of crop yields. Crop yield response functions to salinity usually highlight thresholds within which the loss of production is linear in the salt content [16]. Assuming that irrigation salinity already affects the study area, we consider the following individual damage term : $d Q_{i}$.

Waterlogging. Several authors [12] stress that salinity usually happens in conjunction with waterlogging, and that these phenomena have an impact of crop yield both jointly and in isolation. In this study, we consider that the interactions between these two mechanisms are accommodated by the stock dynamics; hence we formulate the following 'isolated' damage from waterlogging : $f \frac{X^{2}}{2}$.

Salty discharge. Increased discharge of salty water into the surface system results from increased inflows to the groundwater stock. They have consequences on a range of factors, ecological but also linked to human activities. They enter the policy maker's utility function, as a function of the salt content of the discharge : $D \frac{\left(\delta S^{G} X\right)^{2}}{2}$.

3.1.4. Benefit function. We analyze profit-maximizer agents, who use water for irrigation purposes and are affected by soil salinity and waterlogging. They maximize their utility by respect to the use of input (irrigation water) only. In other words, we do not consider irrigation technology as a decision variable. Each technology is associated with an efficiency $\beta$. It impacts on the definition of water remaining in the root zone and percolating. The agents are assumed price-takers with respect to the price of water $p_{e}$ and the price of their production $p$. Their utility function is defined as follows :

$$
B_{i}\left(u_{i}, Q_{i}, X\right)=p\left(a+b u_{i}-c \frac{u_{i}^{2}}{2}-d Q_{i}-f \frac{X^{2}}{2}\right)-p_{e} u_{i}
$$

3.2. Comparison of socially optimal and individual programs. Suppose the existence of a policy maker who wishes to attain an optimal management of a catchment affected by irrigation-induced salinity. He accounts for both on-site and off-site impacts. His maximisation program is as follows :

$$
\max _{u_{i} \geq 0} \int_{0}^{\infty}\left(\sum_{i=1}^{n} B_{i}\left(u_{i}, Q_{i}, X\right)-D\left(S^{G} \delta X\right)\right) e^{-r t} . d t \text { subject to : }
$$

$$
\dot{X}=\sum_{i=1}^{n} P_{i}-(\delta+\gamma) X
$$




$$
\dot{Q}_{i}=S^{W} U_{i}+\gamma S^{G} X-\frac{1-\beta}{\beta} Q_{i}, \forall i .
$$

In this section, we compare the resulting irrigation and stock paths with the ones obtained when analyzing the case of individual agents. We carry out the analysis of individual agents' decision making process in the context of a $n$-player non-cooperative dynamic game. In this paper we only consider one informational structure, the open-loop one. This corresponds to a situation where the agents' decisions at $t$ depend on $t$ and on the initial levels of the stocks. Resulting Open-Loop Nash Equilibria (OLNE) correspond to an infinite period of commitment [8], as the agents commit themselves to a particular water input path, and do not respond to observed variations of the state variable. In the context of irrigation-induced salinity, this would mean that agents do not have access to sufficient information about the groundwater level, which is the case in numerous unmonitored catchments. The individual program is :

$$
\max _{u_{i} \geq 0} \int_{0}^{\infty} B_{i}\left(u_{i}, Q_{i}, X\right) e^{-r t} . d t \text { subject to equations (14) and (15). }
$$

Comparisons between the socially optimum and individual paths are summed up in Propositions 1, 2 and 3.

Proposition 1. There exists an equilibrium to each program (policy maker, open loop agents) with the saddle point property.

Proof. The resolution process follows Dockner [7] who provides an analysis of the local stability of two state variables optimal control problems. Indeed, we consider homogeneous agents, and thus homogeneous salt stocks, the regulator de facto accounts for two state variables. Consequently $\gamma_{i}=\gamma / n, \forall i$. We are seeking to verify if the sufficient and necessary conditions for a unique equilibrium with the saddle point property hold. We provide the full derivation for the social optimum case ${ }^{5}$; refer to Appendix 2 for the open loop case.

The Hamiltonian writes as follows :

$$
\begin{aligned}
H^{s o}=\sum_{i=1}^{n} & B_{i}\left(u_{i}, Q_{i}, X\right)-D\left(\delta S^{G} X\right) \\
& \quad+\lambda^{s o}\left[\sum_{i=1}^{n}(1-\beta) u_{i}-(\delta+\gamma) X\right]+\sum_{i} \mu_{i}^{s o}\left[S^{W} u_{i}+\gamma_{i} S^{G} X-Q_{i} \frac{1-\beta}{\beta}\right],
\end{aligned}
$$

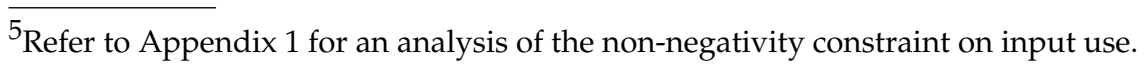


where $\lambda^{s o}$ and $\mu_{i}^{s o}$ are the costate variables associated with groundwater and salinity ${ }^{6}$. Equations (16) to (18) constitute the first-order conditions to the socially optimum program :

$$
\begin{aligned}
& \frac{\partial H^{s o}}{\partial u_{i}}=p \frac{\partial F}{\partial u_{i}}-p_{e}+\lambda^{s o}(1-\beta)+\mu_{i}^{s o} S^{W}, \\
& \lambda^{s o}-r \lambda^{s o}=-\frac{\partial H^{s o}}{\partial X}=X\left(n p f+D \delta S^{G}\right)-\mu_{i}^{s o} \gamma S^{G}+\lambda^{s o}(\delta+\gamma), \\
& \mu_{i}^{s o}-r \mu_{i}^{s o}=-\frac{\partial H^{s o}}{\partial Q_{i}}=-p \frac{\partial F}{\partial Q_{i}}+\mu_{i}^{s o} \frac{1-\beta}{\beta} .
\end{aligned}
$$

Rearrange equations (16)-(17)-(18) to obtain equation (16.a)-(17.a)-(18.a). Equation (16.a) exhibits the standard equality of marginal benefits and costs associated with using one more unit of irrigation water :

$$
p\left(b-c u_{i}\right)=p_{e}-\lambda^{s o}(1-\beta)-\mu^{i} S^{W}
$$

The marginal costs consist of the direct cost of buying water, the indirect cost of an additional unit of water percolating and the indirect cost of salty water brought by irrigation and remaining in the rootzone.

(17.a) $\lambda^{\dot{s o}}=(r+\delta+\gamma) \lambda^{s o}+\left(D \delta S_{G}+n p f\right) X-\mu_{i}^{s o} \gamma S^{G}$.

Equation (17.a) simply illustrates that the costate variable associated with the watertable dynamics must increase at the rate of $(r+\delta+\gamma)$ and taking account of the downstream damage (in terms of discharge of salty water in the surface system) and of the salts brought upwards by capillary rise.

$$
\text { (18.a) } \mu_{i}^{s o}=\left(r+\frac{1-\beta}{\beta}\right) \mu_{i}^{s o}+d p \text {. }
$$

In equation (18.a), the costate variable associated with the salt accumulation in the rootzone increases at the rate $\left(r+\frac{1-\beta}{\beta}\right)$ and taking account of the damage on production due to salts in the rootzone. From equation (16.a), we get :

$$
u_{i}=\left[\lambda^{s o}(1-\beta)+\mu_{i}^{s o} s^{W}+p b-p_{e}\right] / p c .
$$

Replace $u_{i}$ by this expression in equations (14) and (15) and add equations (17.a) and (18.a) to form the Modified Hamiltonian Dynamic System (MHDS) :

$$
\left(\begin{array}{c}
\dot{X} \\
\lambda^{s o} \\
\dot{Q}_{i} \\
\mu^{s o}
\end{array}\right)=\left(\begin{array}{cccc}
-\delta-\gamma & \frac{n(1-\beta)^{2}}{p c} & 0 & \frac{n S^{W}}{p c}(1-\beta) \\
D \delta S^{G}+n p f & r+\delta+\gamma & 0 & -\gamma S^{G} \\
\gamma_{i} S^{G} & \frac{S^{W}}{p c}(1-\beta) & \frac{\beta-1}{\beta} & \frac{s^{W 2}}{p c} \\
0 & 0 & 0 & r+\frac{1-\beta}{\beta}
\end{array}\right) \cdot\left(\begin{array}{c}
X \\
\lambda^{s o} \\
Q_{i} \\
\mu^{s o}
\end{array}\right)+\left(\begin{array}{c}
n(1-\beta) \frac{p b-p_{e}}{p c} \\
0 \\
S^{W} \frac{p b-p_{e}}{p c} \\
d p
\end{array}\right)
$$

\footnotetext{
${ }^{6}$ It is assumed that the conditions for an interior solution hold, so that the non negativity constraint on $u_{i}$ doesn't bing. Refer to Appendix 5 for an analysis of non negativity conditions.
} 
Let $J$ stand for the matrix with typical elements $\partial u / \partial v$ with $u, v \in\left\{X, Q_{i}, \lambda, \mu_{i}\right\}$ and $C$ for the constants matrix. Define $K$ as follows :

$$
K=\left|\begin{array}{ll}
\frac{\partial \dot{X}}{\partial X} & \frac{\partial \dot{X}}{\partial \lambda} \\
\frac{\partial \dot{\lambda}}{\partial X} & \frac{\partial \dot{\lambda}}{\partial \lambda}
\end{array}\right|+\left|\begin{array}{ll}
\frac{\partial \dot{Q}_{i}}{\partial Q_{i}} & \frac{\partial \dot{Q}_{i}}{\partial \mu_{i}} \\
\frac{\partial \dot{\mu}_{i}}{\partial Q_{i}} & \frac{\partial \dot{\mu}_{i}}{\partial \mu_{i}}
\end{array}\right|+2\left|\begin{array}{cc}
\frac{\partial \dot{X}}{\partial Q_{i}} & \frac{\partial \dot{X}}{\partial \mu_{i}} \\
\frac{\partial \dot{\lambda}}{\partial Q_{i}} & \frac{\partial \dot{\lambda}}{\partial \mu_{i}}
\end{array}\right|
$$

The conditions $K<0$ and $0<\operatorname{det} J<\left(\frac{K}{2}\right)^{2}$ are necessary and sufficient to ensure the saddlepoint property [7].

Let $z_{1}^{s o}=\frac{1-\beta}{\beta}\left(r+\frac{1-\beta}{\beta}\right)>0$ and $z_{2}^{s o}=(\delta+\gamma)(r+\delta+\gamma)+\frac{n}{p c}(1-\beta)^{2}\left(D \delta S^{G}+n p f\right)>0$.

Then $K=-z_{1}^{s o}-z_{2}^{s o}<0$, which ensures that the first condition is met. Also, $\operatorname{det} J=$ $z_{1}^{s o} z_{2}^{s o}>0$. Then $\left(\frac{K}{2}\right)^{2}-\operatorname{det} J=\frac{1}{4}\left(z_{1}^{s o}+z_{2}^{s o}\right)^{2}-z_{1}^{s o} z_{2}^{s o}=\frac{1}{4}\left(z_{1}^{s o}-z_{2}^{s o}\right)^{2}>0$. Hence the second condition is met.

Proposition 2. The steady state groundwater and rootzone salt stocks are higher in the open loop case than in the socially optimum case.

Proof. The value of the steady state variables is obtained after recognizing that the steady state, the following relation holds : $Z^{\infty}=-J^{-1}$. $C$ where $Z^{\infty}$ stands for the matrix with typical elements $v^{\infty}, v \in\left\{X, Q_{i}, \lambda, \mu_{i}\right\}$. Computations with the Maple software give the following steady state values of the groundwater (respectively rootzone salt) stocks and input use paths, for $m=\{s o, o l\}$ :

$$
\begin{aligned}
X_{\infty}^{m} & =\frac{1}{z_{2}^{m}}\left[M_{1}+\mu_{\infty} M_{2}^{m}\right] \frac{n(1-\beta)}{p c}, \\
Q_{\infty}^{m} & =\frac{\beta}{1-\beta}\left[\gamma_{i} S^{G} X_{\infty}^{m}+S^{W} u_{\infty}^{m}\right], \\
u_{\infty}^{m} & =\frac{1}{p c}\left[p b-p_{e}+\mu_{\infty}^{m} S^{W}+\lambda_{\infty}^{m}(1-\beta)\right] .
\end{aligned}
$$

Refer to Appendix 3 for the values of the parameters. Then $z_{2}^{s o}>z_{2}^{o l}$ and $M_{2}^{s o}>M_{2}^{o l}$ imply $X_{\infty}^{s o}<X_{\infty}^{o l}$. It is also straightforward to note that $\left|\lambda_{\infty}^{o l}\right|<\left|\lambda_{\infty}^{s o}\right|$. Also, from equation (21), we know that $u_{\infty}^{o l}>u_{\infty}^{s o}$, consequently $Q_{\infty}^{o l}>Q_{\infty}^{s o}$.

The steady state value of the costate variable associated with individual salt stock, $\mu_{\infty}$, is negative, and has the same value in the open loop and social optimum cases. It depends on the extent of individual damage and on an environmental discount rate, $r+\frac{(1-\beta)}{\beta}$. It is the sum of the usual discount rate and of the discharge rate associated with the stock of salt. A higher discount rate induces a lower value of $\left|\mu_{\infty}\right|$ : when the future counts less, or when the natural discharge capacity is high, the stock's shadow price decreases. The contrary happens when the individual damage term increases.

The steady state groundwater stock is related to the extent of damage due to its accumulation. The higher the damage, the smaller the steady state groundwater stock. The 
steady state salt stock accounts for the various types of damages.

Proposition 3. Open loop agents induce a higher accumulation of groundwater than what is socially optimum, which indirectly leads to a higher accumulation of the root-zone salts.

Proof. To derive the stock and input paths, the resolution method is a follows. We find the eigenvalues of matrix $\mathrm{J}$, and choose the negative ones $w_{j}, j \in\{1,2\}$ to ensure stability. Then we compute the associated eigenvectors $w_{j v}, v \in\left\{X, Q_{i}, \lambda, \mu_{i}\right\}$. The solutions are of the following form [3]:

$$
\varphi\left(v, v_{\infty}, v_{0}, t\right)=v_{\infty}+c_{1} w_{1 v} e^{w_{1} t}+c_{2} w_{2 v} e^{w_{2} t} \text { with } v \in\left\{X, Q_{i}, \lambda, \mu_{i}\right\} .
$$

The groundwater, rootzone salinity and water input paths are, for $m=\{s o, o l\}$ :

$$
\begin{aligned}
& X^{m}(t)=X_{\infty}^{m}+\left(X_{0}-X_{\infty}^{m}\right) e^{w_{1}^{m} t}, \\
& Q_{i}^{m}(t)=Q_{\infty}^{m}+\left(Q_{0}-Q_{\infty}^{m}\right) e^{w_{2}^{m} t}+\frac{X_{0}-X_{\infty}^{m}}{w_{1 X}^{m}}\left(e^{w_{1}^{m} t}-e^{w_{2}^{m} t}\right), \\
& u^{m}(t)=\lambda^{m}(t) \frac{1-\beta}{p c}+\mu_{\infty} \frac{S^{W}}{p c}+\frac{p b-p_{e}}{p c} .
\end{aligned}
$$

Refer to Appendix 4 for the values of the parameters.

Assuming that the initial groundwater stock is below the steady state, so that the issue at stake is an accumulation problem, the groundwater stock path is monotonic, and increasing from $X_{0}$ to $X_{\infty}^{m}$ at the rate $w_{1}^{m}$. Then, as $w_{1}^{s o}<w_{1}^{o l}$, it follows that open-loop agents not only accumulate more groundwater, they also accumulate faster than what is socially optimal.

The salt stock's behavior depends on two phenomena. The first part of the RHS of equation (23), $Q_{\infty}^{m}+\left(Q_{0}-Q_{\infty}^{m}\right) e^{w_{2}^{m} t}$, is an autonomously increasing part, in the sense that it is not directly affected by the collective groundwater stock. The second part of the RHS shows the correlation between the individual and the collective stocks. The sign of the interaction depends on three terms. First, $X_{0}-X_{\infty}^{m}<0$ in cases of accumulation. Then, $e^{w_{1}^{m} t}-e^{w_{2}^{m} t}>0$, as the exponential function is monotonously increasing, and because the following applies :

$$
w_{1}^{m}>w_{2}^{m} \Rightarrow 1-\beta>-\beta w_{1}^{m} \Rightarrow 1-\beta>-\beta r / 2 \text {, as } w_{1}^{m}<r / 2 .
$$

Third, the sign of $w_{1 X}$ is a priori undetermined. It can be shown that its numerator is positive, however its denominator is more difficult to assess. It comprises two terms, $n(1-\beta) \gamma S^{G}$ and $S^{w}\left(w_{1}^{m}+\delta+\gamma\right)$; the term $w_{1}^{m}+\delta+\gamma$ illustrates a tradeoff between dissipating and accumulating features of the groundwater stock, the greater the accumulation rate, the lower $w_{1}^{m}+\delta+\gamma$, hence the greater $w_{1 X}$.

After investigating these benchmark cases, in the next section we address the design of taxes to attain the optimal solution. We consider various taxes, basing either on the 
amount of irrigation input, the groundwater stock, or both. We also consider two definitions of optimality : the policy maker may want irrigators to behave optimally along the whole paths, or only to reach the optimal steady state.

\section{Designing OPtimal WATER tAXES}

In this section, we assess the potential of various dynamic taxes to induce the agents to take optimal decisions. We consider the following general scheme :

$$
\tau\left(U_{i}, X\right)=\tau_{1} u_{i}+\tau_{2} u_{i} X+\tau_{3} X
$$

Such a formulation encompasses the various types of dynamic taxes that have been analysed in the literature; $\tau_{2}=\tau_{3}=0$ is a time-independant water tax, $\tau_{1}=\tau_{2}=0$ is a linear state-dependent ambient tax [11] and $\tau_{3}=0$ is a stock dependant tax [1]. We focus on two potential tax bases, the amount of irrigation water $u_{i}$ and the groundwater stock. Implicit in this formulation is the consideration that individual salt stocks are not realistically observable by the regulator, or only at a prohibitive cost.

Through an analysis of the general scheme, we highlight the differentiated impacts of the parameters $\tau_{1}, \tau_{2}$ and $\tau_{3}$ on the accumulation paths and the steady state values. Then we compute the optimal parameters, when optimality is required at the steady state only, or along the whole time horizon.

4.1. The general taxation scheme. Consider an agent, following an open loop strategy, subject to a taxation scheme as described in equation (25). His maximisation problem is :

$$
\max _{u_{i}} B_{i}\left(u_{i}, Q_{i}, X\right)-\tau\left(u_{i}, X\right) \text { subject to equations (14) and (15). }
$$

Proposition 4. The introduction of taxes based on the individual use of water and/or the level of groundwater does not alter the saddle point property of the equilibrium.

Proof. Using the same notations as in the previous section, the MHDS writes as $Z_{\infty}=$ $J^{\tau} Z+C^{\tau}$ with $^{7}$ :

$$
J^{\tau}=\left(\begin{array}{cccc}
A\left(\tau_{2}\right) & B & 0 & C \\
D\left(\tau_{2}\right) & E\left(\tau_{2}\right) & 0 & F\left(\tau_{2}\right) \\
G\left(\tau_{2}\right) & H & I & J^{\prime} \\
0 & 0 & 0 & K^{\prime}
\end{array}\right) \text { and } C^{\tau} t=\left(\begin{array}{c}
L\left(\tau_{1}\right) \\
M\left(\tau_{1}, \tau_{2}, \tau_{3}\right) \\
N\left(\tau_{1}\right) \\
P
\end{array}\right)
$$

The necessary and sufficient conditions to ensure the saddle point property are $K<0$ and $(K / 2)^{2}-\operatorname{det} J>0, K$ being defined as in the previous sections. Define :

$$
\begin{aligned}
& z_{1}^{\tau} t=-I K^{\prime}>0, \\
& z_{2}^{\tau}=-A\left(\tau_{2}\right) E\left(\tau_{2}\right)+D\left(\tau_{2}\right) B>0
\end{aligned}
$$

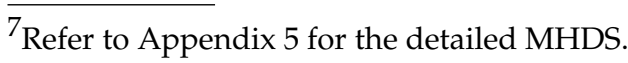


Then $K=-z_{1}^{\tau}-z_{2}^{\tau}<0$ and $(K / 2)^{2}-\operatorname{det}\left(J^{\tau}\right)=z_{1}^{\tau} z_{2}^{\tau}>0$. Both conditions are satisfied, ensuring the saddle point property.

Proposition 5. Each tax parameter, associated to individual input use $\left(\tau_{1}\right)$, collective groundwater stock $\left(\tau_{3}\right)$ or a mix of both $\left(\tau_{2}\right)$, impacts on the steady state values of the stocsk and input choice. However, only $\tau_{2}$ affects the groundwater stock accumulation rate, thus indirectly the rootzone salt stock's speed of accumulation.

Proof. Applying the same method as in the previous cases, we derive the following steadystate values of the variables :

$$
\begin{aligned}
X_{\infty}^{\tau} & =\frac{1}{z_{2}^{\tau}}\left[M_{1}^{\tau}\left(\tau_{1}, \tau_{3}\right)+M_{2}^{\tau} \mu_{\infty}\right], \\
Q_{\infty}^{\tau} & =\frac{\beta}{\beta-1}\left[X_{\infty}^{\tau} \gamma S^{G}+S^{W} u_{\infty}^{\tau}\right], \\
u_{\infty}^{\tau} & =\frac{1}{p c}\left[p b-p_{e}-\tau_{1}-\tau_{2}+\mu_{\infty} S^{W}+\lambda_{\infty}^{\tau}(1-\beta)\right], \\
\lambda_{\infty}^{\tau} & =\frac{-1}{z_{2}^{\tau}}\left[M_{3}^{\tau}\left(\tau_{1}, \tau_{2}, \tau_{3}\right)-\mu_{\infty} M_{4}^{\tau}\left(\tau_{2}\right)\right], \\
\mu_{\infty}^{\tau} & =\mu_{\infty}^{o l}=\mu_{\infty}^{s o} .
\end{aligned}
$$

Furthermore, the paths are of the same form as in the previous case :

$$
\begin{aligned}
& X^{\tau}(t)=X_{\infty}^{\tau}+\left(X_{0}-X_{\infty}^{\tau}\right) e^{w_{1}^{\tau} t} \\
& \lambda^{\tau}(t)=\lambda_{\infty}^{\tau}+\left(X_{0}-X_{\infty}^{\tau}\right) \frac{w_{12}^{\tau}}{w_{11}^{\tau}} e^{w_{1}^{\tau} t} \\
& Q_{i}^{\tau}(t)=Q_{\infty}^{\tau}+\left(Q_{0}-Q_{\infty}^{\tau}\right) e^{w_{2}^{\tau} t}+\frac{X_{0}-X_{\infty}^{\tau}}{w_{11}^{\tau}}\left(e^{w_{1}^{\tau} t}-e^{w_{2}^{\tau} t}\right), \\
& u_{i}^{\tau}(t)=\lambda^{\tau}(t) \frac{1-\beta}{p c}-X^{\tau}(t) \frac{\tau_{2}}{p c}+\mu_{\infty} \frac{S^{W}}{p c}+\frac{p b-p_{e}-\tau_{1}}{p c} .
\end{aligned}
$$

To assess the impact of the various tax parameters on the steady state values, we derive the following expressions: $\partial M_{1}^{\tau} / \partial \tau_{1}<0 \partial M_{1}^{\tau} / \partial \tau_{3}<0$. As $z_{2}^{t}>0$ and $\partial z_{2}^{t} / \partial \tau_{2}>0$, the steady state groundwater stock is clearly negatively affected by the individual, collective and mixed tax parameters. However the impact on the associated co-state variable is not analytically tractable. Indeed, the following relationships apply:

$$
\frac{\partial M_{3}^{\tau}}{\partial \tau_{1}}>0, \frac{\partial M_{3}^{\tau}}{\partial \tau_{2}}<0, \frac{\partial M_{3}^{\tau}}{\partial \tau_{3}}<0 \text { and } \frac{\partial M_{4}^{\tau}}{\partial \tau_{2}} \text { undet. }
$$

The eigenvalues, which dictate the speed of accumulation, are :

$$
w_{1}^{\tau}=\left[A\left(\tau_{2}\right)+E\left(\tau_{2}\right)\right] / 2-\sqrt{\left(A\left(\tau_{2}\right)-E\left(\tau_{2}\right)\right)^{2}+4 D\left(\tau_{2}\right) B} / 2<0 \text { and } w_{2}^{\tau}=I<0
$$

It is straightforward to notice that none of the taxes under study has an impact on the value of $w_{2}^{\tau}$. However, $\tau_{2}$ does appear in $w_{1}^{\tau}$. The first order derivative of $w_{1}^{\tau}$ with respect 
to the relevant tax parameter are as follows :

$$
\begin{aligned}
& \frac{\partial w_{1}^{\tau}}{\partial \tau_{2}}=1 / 2\left[(1-\beta)(1-n)-1 / 2 \frac{M_{6}^{\tau^{\prime}}\left(\tau_{2}\right)}{\sqrt{M_{6}^{\tau}\left(\tau_{2}\right)}}\right]<0, \\
& \text { with } M_{6}^{\tau}\left(\tau_{2}\right)=\tau_{2}^{2} \frac{(n-1)^{2}(1-\beta)^{2}}{2 p c}+\tau_{2}(n+1)(1-\beta)(r+2 \delta+2 \gamma)+C T E .
\end{aligned}
$$

These derivations confirm the insights from the general case study, that is that the mixed tax parameter $\tau_{2}$ has a special status in the sense that it impacts on both the steady state values and the accumulation rate. Next we provide the full derivation of the optimal tax parameters, and make use of this property of $\tau_{2}$ to induce the agents to follow the optimal path along the whole time horizon. In a first step, we assume that the regulator requires the agents to comply with the optimal level of groundwater at the steady state. In this regard, it constitutes an application of Xepapadeas' [21] definition of an efficient scheme ${ }^{8}$. In a second step, we assume that the regulator requires a more standard definition of efficiency, which the is requirement that the agents comply with the optimal accumulation path along the whole time horizon.

\subsection{Optimal taxes parameters : steady state only.}

Proposition 6. In order to induce the agents following open-loop strategies to reach the optimal steady state, the regulator can either use a pure ambient tax or a time-independent input tax.

Proof. We solve the system $\left\{X_{\infty}^{S O}=X_{\infty}^{T}, Q_{\infty}^{S O}=Q_{\infty}^{T}\right\}$ with respect to different combinations of the tax parameters, as shown in the following table.

\begin{tabular}{lll||lll}
\hline Case & Solve w.r.t. & Opt. Par. & Case & Solve w.r.t. & Opt. Par. \\
\hline $\mathbf{a}$ & $\tau_{1}, \tau_{2}, \tau_{3}$ & $\tau_{3}\left(\tau_{1}, \tau_{2}\right)$ & $\mathbf{e}$ & $\tau_{3}\left[\tau_{1}=\tau_{2}=0\right]$ & $\hat{\tau_{3}}$ \\
$\mathbf{b}$ & $\tau_{1}, \tau_{2}\left[\tau_{3}=0\right]$ & $\tau_{1}\left(\tau_{2}\right)$ & $\mathbf{f}$ & $\tau_{2}\left[\tau_{1}=\tau_{3}=0\right]$ & $\hat{\tau_{2}}$ \\
$\mathbf{c}$ & $\tau_{1}, \tau_{3}\left[\tau_{2}=0\right]$ & $\tau_{3}\left(\tau_{1}\right)$ & $\mathbf{g}$ & $\tau_{1}\left[\tau_{2}=\tau_{3}=0\right]$ & $\hat{\tau}_{1}$ \\
$\mathbf{d}$ & $\tau_{2}, \tau_{3}\left[\tau_{1}=0\right]$ & $\tau_{3}\left(\tau_{2}\right)$ & & & \\
\hline \multicolumn{6}{c}{ TABLE 1. Optimal steady state tax rates } \\
\hline
\end{tabular}

When any of the parameters associated with individual performance is equal to zero (cases $\mathbf{c}$ and $\mathbf{d}$ ), then the optimal tax takes the form of a pure ambient tax, with a tax rate equal to $\hat{\tau_{3}}$. Case a constitutes a case of over-information. Indeed, when the three parameters are available, any choice of $\tau_{1}$ and $\tau_{2}$ and associated $\tau_{3}\left(\tau_{1}, \tau_{2}\right)$ allows attaining the optimal steady state (in particular when $\tau_{1}$ and $\tau_{2}$ are set equal to zero). When the pure collective parameter $\tau_{3}$ is not available, the optimal tax is an input tax (cases $\mathbf{b}$ and g).

\footnotetext{
${ }^{8} \mathrm{~A}$ similar approach in the context of public good provision is provided by Fershtman and Nitzan [10].
} 
For the sake of readability we don't include the analytical expressions of the taxes in this paper ${ }^{9}$. However we provide some numerical illustrations in section 5.

\subsection{Optimal taxes parameters : along the whole time horizon.}

Proposition 7. To induce open agents to take optimal decisions along the whole time horizon, the regulator needs to have recourse to a mixed tax, based on parameter $\tau_{2}$, and one of the purely collective $\left(\tau_{3}\right)$ or individual $\left(\tau_{1}\right)$ ones.

Proof. We follow the methodology used by [1]. We seek to have $u^{s o}(X)$ and $\tau^{*}\left(\tau_{1}, \tau_{2}, \tau_{3}\right)$ satisfy the first order conditions of open loop agents subject to the general tax scheme ${ }^{10}$. In other words, we want to induce open loop agents, subject to the optimal tax scheme $\tau^{*}\left(\tau_{1}, \tau_{2}, \tau_{3}\right)$, to use the optimal reaction function $u^{s o}(X)$. Remember the following socially optimum first order condition on input use and collective stock co-state variable path :

$$
\begin{aligned}
& p\left(b-c u_{i}\right)=p_{e}-\lambda^{s o}(1-\beta)-\mu^{i} S^{W}, \\
& \lambda^{s o}=\lambda_{\infty}^{s o}+\left(X_{0}-X_{\infty}^{s o}\right) \frac{w_{1 \lambda}^{s o}}{w_{1 X}^{s o}} e^{w_{1}^{s o} t}=\lambda_{\infty}^{s o}+\frac{w_{1 \lambda}^{s o}}{w_{1 X}^{s o}}\left[X-X_{\infty}^{s o}\right] .
\end{aligned}
$$

Replace $\lambda^{s o}$ with its value taken from (17), and derive (16) with respect to time to obtain :

$$
p c u^{\prime}(X) \dot{X}=-\tau_{2} \dot{X}+\dot{\lambda}^{\tau}(1-\beta) .
$$

Knowing that :

$$
\begin{aligned}
\dot{\lambda^{\tau}} & =(r+\delta+\gamma) \lambda^{t}+p f X+\tau_{2} u_{i}+\tau_{3}-\mu_{\infty} \gamma S G, \\
\lambda^{\tau} & =\lambda_{\infty}^{\tau}+\frac{w_{1 \lambda}^{\tau}}{w_{1 X}^{\tau}}\left[X-X_{\infty}^{\tau}\right],
\end{aligned}
$$

and rearranging (26), we obtain a first order polynom of $X$ :

$$
C_{1}\left(\tau_{2}\right) X+C_{0}\left(\tau_{1}, \tau_{2}, \tau_{3}\right)=0, \forall X \Rightarrow C_{1}\left(\tau_{2}\right)=0 \text { and } C_{0}\left(\tau_{1}, \tau_{2}, \tau_{3}\right)=0 .
$$

Solutions are obtained for the following combinations of parameters : $\left\{\tau_{1}, \tau_{2}\right\}$ and $\left\{\tau_{2}, \tau_{3}\right\}$. This confirms the needs for a stock-dependant mixed tax to induce optimality along the whole time horizon. A standard input tax, or a pure ambient tax, is not sufficient to induce the agents to irrigate in a optimal way, and consequently accumulate optimaly both individual rootzone salt stocks, and the collective groundwater stock.

\section{NUMERICAL ILLUSTRATIONS}

The values of the parameters in use in the simulations are as follows : $\delta=0.01, \gamma=$ $0.0001, X_{0}=0, S^{G}=5 E C, B_{i}\left(u_{i}, Q_{i}, X\right)=0.05+1.95 u_{i}-\frac{0.80}{2} u_{i}^{2}-0.01 Q_{i}-\frac{0.0001}{2} X^{2}$, $\beta=0.6, S^{W}=1.5 E C, Q_{0}=0, p=5, p_{e}=1, r=0.04$ and $n=10$. Figure 1 illustrates the variation between individual and socially optimal decisions through its impact on individual and collective stock accumumation. Figure 2 illustrates the differentiated impact

\footnotetext{
${ }^{9}$ They are available on request.

${ }^{10}$ Refer to Appendix 5.
} 
of various steady state taxes on the accumulation of the stocks.
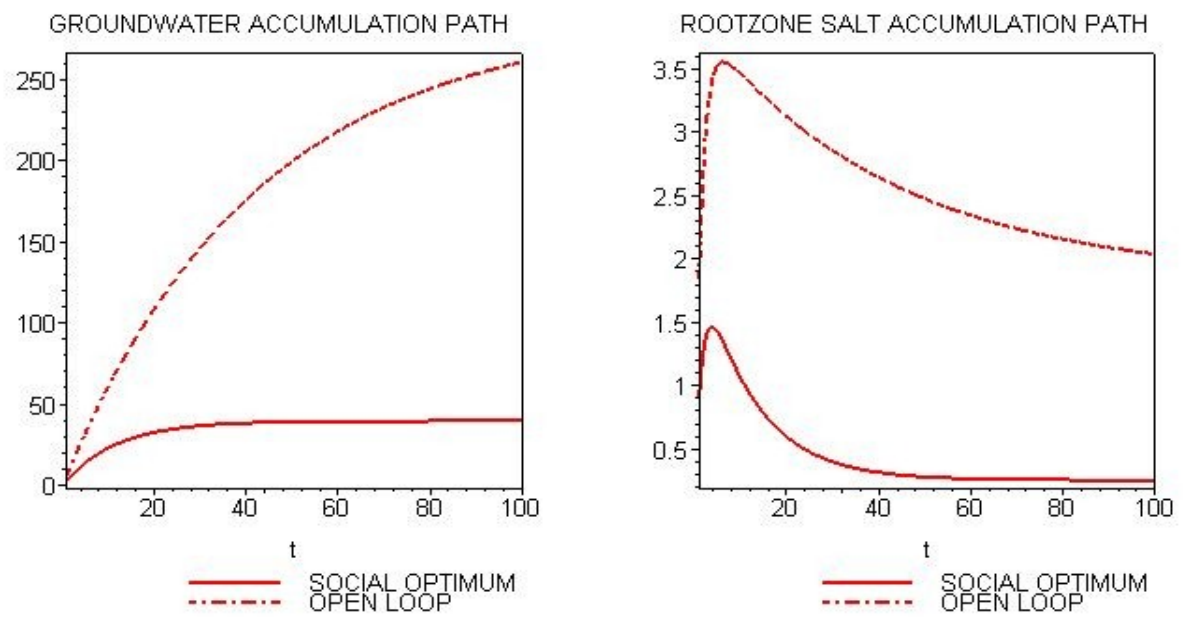

FIGURE 1. Comparison social optimum and open loop paths - groundwater and rootzone salts stocks
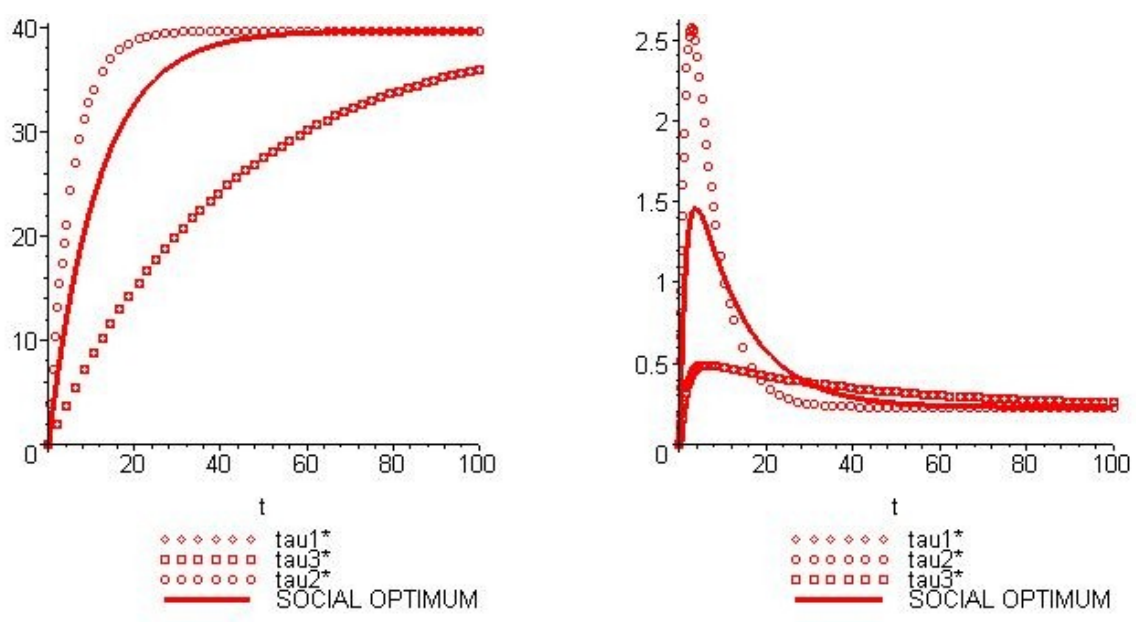

FIGURE 2. Impact of various steady state taxes on groundwater/rootzone stock path 


\section{CONCLUDING REMARKS}

This paper examined the problem of designing optimal water taxes for irrigators in the context of irrigation-induced salinity, when a collective groundwater stock interacts with individual rootzone salt stocks. In this regard, the context constitutes a special case of correlated stock externalities, in which the stocks are positively correlated and it is the collective stock that impacts on the individual stocks.

First we compared socially optimal decisions and decisions taken by agents following open-loop strategies and showed that in the latter case, groundwater is accumulated more, and more rapidly. This provides a rationale for policy intervention. Then we addressed the impact of a general taxation scheme, based on both individual performance (individual stock, input use) and collective performance (groundwater stock). We highlighted some features of the taxation schemes, in particular the need for a mixed tax parameter to induce the agents to optimally correct their accumulation rate. Finally, we derived the optimal parameters in various understandings of optimality, at the steady state only or along the whole time horizon. We showed that, if a pure ambient tax is sufficient in the former case, it is necessary to have recourse to a mixed tax parameter to ensure optimality of the accumulation paths.

The interaction between the stocks is quite particular in the setting of irrigation-induced salinity. First, the correlation is positive, so that an increase in the level of groundwater leads to an increase of rootzone salts. Second, in this paper we only considered that the global stock impacts on the local ones. Indeed, in a more realistic, hence complex, model of irrigation-induced salinity, it would be necessary to describe a stock of salt and a stock of water for each reservoir at stake (river system, watertable, individual rootzones). Then the individual salt stocks would impact on the global one. However, multiple state variables problems rapidly become not analytically tractable, hence our choice to focus on the main sources of damage : rootzone salinity and groundwater level. Consequently, the taxes' impacts on the individual stocks is of the same order as their impacts on the collective stock.

Further research could include considering agents following feedback strategies, as policy instruments based on a measure of collective performance typically induce strategic interactions between the agents. Such strategic interactions could alter the efficiency of the taxation schemes analyzed here.

\section{REFERENCES}

[1] H. Benchekroun and N. van Long. Efficiency inducing taxation for polluting oligopolists. Journal of Public Economics, 70:325-342, 1998.

[2] O. Bergland, R. Ready, and E. Romstad. Differentiating at the Speed of Light in a Universe of Trees and Moose, pages 202-224. The Theory and Practice of Environmental and Resource Economics - Essays in Honor of Karl-Gustaf Löfgren. Edward Elgar, Cheltenham, UK. 
[3] C.A. Bond and Y.H. Farzin. Multiple nutrient stocks and sustainable agriculture. Paper presented to the 2004 EAERE conference, Budapest, 25-28 June, 2004.

[4] J.I. Bulow, J.D. Geanakoplos, and P.D. Klemperer. Multimarket oligopoly : Strategic substitutes and complements. The Journal of Political Economy, 3:488-511, 1985.

[5] A.J. Caplan and E.C.D. Silva. An efficient mechanism to control correlated externalities: redistributive transferts and the coexistence of regional and global pollution permit markets. Journal of Environmental Economics and Management, 49:68-82, 2005.

[6] A.S. Crépin. Management challenges for multiple-species boreal forests. FEEM Working Paper No. 106.2003, 2003.

[7] E. Dockner. Local stability analysis in optimal control problems with two state variables, pages 89-103. Optimal Control Theory and Economic Analysis 2. Elsevier, 1985.

[8] E. J. Dockner, S. Jorgensen, N. Van Long, and G. Sorger. Differential Games in Economics and Management Science. Cambridge University Press, 2000.

[9] Y.H. Farzin and O. Tahvonen. Global carbon cycle and the optimal time path of a carbon tax. Oxford Economic Papers, 48:515-536, 1996.

[10] C. Fershtman and S. Nitzan. Dynamic voluntary provision of public goods. European Economic Review, 35:1057-67, 1991.

[11] R.D. Horan, J.S. Shortle, and D.G. Abler. Ambient taxes when polluters have multiple choices. Journal of Environmental Economics and Management, 36:186-199, 1998.

[12] M.A. Kahlown and M. Azam. Individual and combined effect of waterlogging and salinity on crop yields in the Indus basin. Irrigation and drainage, 51:329-338, 2002.

[13] L. Karp and J. Livernois. On efficiency-inducing taxation for a nonrenewable resource monopolist. Journal of Public Economics, 49:219-239, 1992.

[14] I.D. Ko, H.E. Lapan, and T. Sandler. Controlling stock externalities - flexible versus inflexible pigovian corrections. European Economic Review, 35:1263-1276, 1992.

[15] S. Legras and R. Lifran. Dynamic taxation schemes to manage irrigation induced salinity. Environmental Modeling and Assessment, Forthcoming.

[16] E.V. Maas and G.J. Hoffman. Crop salt tolerance- current assessment. Journal of the Irrigation and Drainage Division, ASCE 103(IR2):115-134, 1977.

[17] D. Ragozin and G. Brown. Harvest policies and nonmarket valuation in a predator-prey system. Journal of Environmental Economics and Management, 12:155-168, 1985.

[18] R.M. Saleth. Towards a new water institution: economics, law, and policy. Econom. Political Wkly, 29(39):A147-A155, 1994.

[19] D.L. Umali. Irrigation-induced salinity: a growing problem for development and the environment. Technical report, The World Bank, Washington, D.C., 1993.

[20] D. Wichelns. An economic model of waterlogging and salinization in arid regions. Ecological Economics, 30(3):475-491, 1999.

[21] A.P. Xepapadeas. Environmental policy design and dynamic nonpoint-source pollution. Journal of Environmental Economics and Management, 23:22-39, 1992.

[22] Z. Yang. Negatively correlated local and global stock externalities: tax or subsidy? Environment and Development Economics, 11:301-316, 2006

\section{Appendix 1 : Non negativity constraint of the optimal input use.}

The condition for an interior solution is to have, $\forall t, u(t)>0$. Remember we have the following irrigation path :

$$
u_{i}^{s o}=\lambda \frac{1-\beta}{p c}+\mu_{\infty} \frac{S^{W}}{p c}+\frac{p b-p_{e}}{p c}
$$


As $\lambda^{s o}$ is a strictly decreasing function of time, so is $u^{s o}(t)$. To ensure that $\forall t, u^{s o}>0$ it is sufficient to show that $u_{\infty}^{s o}>0$.

$$
\begin{aligned}
& u_{\infty}^{s o}=\frac{1}{p c}\left[p b-p_{e}+\mu_{\infty}^{s o} S^{W}+\lambda^{s o}(1-\beta)\right]=\frac{N\left(u_{\infty}^{s o}\right)}{D\left(u_{\infty}^{s o}\right)}, \\
& D\left(u_{\infty}^{s o}\right)=[r \beta+1-\beta]\left[p c(r+\delta+\gamma)(\delta+\gamma)+n^{2} p f(1-\beta)^{2}+n D S^{G} \delta(1-\beta)^{2}\right]>0, \\
& N\left(u_{\infty}^{s o}\right)=\left(p b-p_{e}\right)(r+\delta+\gamma)(\delta+\gamma)(r \beta+1-\beta)-d p \beta(\delta+\gamma)\left[n S^{G} \gamma(1-\beta)+S^{W}(r+\delta+\gamma)\right] .
\end{aligned}
$$

Consequently, $u_{\infty}^{s o}>0$ if its numerator is positive, which amounts to :

$$
\frac{p b-p_{e}}{d p}\left(r+\frac{1-\beta}{\beta}\right)>\frac{n S^{G} \gamma(1-\beta)}{r+\delta+\gamma} .
$$

\section{Appendix 2 : Open-loop case : derivation of the paths and non negativity constraint}

The individual maximisation program is now :

$$
\max B_{i}\left(u_{i}, Q_{i}, X\right) \text { subject to (14), (15) and } u_{i} \geq 0 \text {. }
$$

Supposing we are in the conditions of an interior solution, the MHDS is :

$$
\left(\begin{array}{c}
\dot{X} \\
\dot{\lambda} \\
\dot{Q}_{i} \\
\dot{\mu}
\end{array}\right)=\left(\begin{array}{cccc}
-\delta-\gamma & \frac{n(1-\beta)^{2}}{p c} & 0 & \frac{S^{W}}{p c}(1-\beta) \\
p f & r+\delta+\gamma & 0 & -\gamma S^{G} \\
\gamma_{i} S^{G} & \frac{S^{W}}{p c}(1-\beta) & \frac{\beta-1}{\beta} & \frac{S^{W 2}}{p c} \\
0 & 0 & 0 & r+\frac{1-\beta}{\beta}
\end{array}\right) \cdot\left(\begin{array}{c}
X \\
\lambda \\
Q_{i} \\
\mu
\end{array}\right)+\left(\begin{array}{c}
n(1-\beta) \frac{p b-p_{e}}{p c} \\
0 \\
S^{W \frac{p b-p_{e}}{p c}} \\
d p
\end{array}\right)
$$

The resolution process is the same as in the previous case. Let $z_{1}^{\prime}=\frac{1-\beta}{\beta}\left(r+\frac{1-\beta}{\beta}\right)>0$ and $z_{2}^{\prime}=(\delta+\gamma)(r+\delta+\gamma)+\frac{n}{p c} f p(1-\beta)^{2}>0$. The rest of the demonstration follows.

To derive the conditions for the nonnegativity of the control variables, the same methodology as in the optimal case.

$$
\begin{aligned}
& D\left(U_{\infty}^{o l}\right)=[r \beta+1-\beta]\left[p c(r+\delta+\gamma)(\delta+\gamma)+n p f(1-\beta)^{2}\right]>0, \\
& N\left(U_{\infty}^{o l}\right)=\left(p b-p_{e}\right)(r+\delta+\gamma)(\delta+\gamma)(r \beta+1-\beta) \\
& -d p \beta\left[S^{G} c \gamma(1-\beta)(\delta+\gamma)+S^{W}\left(f(n-1)(1-\beta)^{2}+c(r+\delta+\gamma)(\delta+\gamma)\right],\right. \\
& u_{\infty}^{o l}>0 \Rightarrow \frac{p b-p_{e}}{d p}\left(r+\frac{1-\beta}{\beta}\right)>S^{W}\left[1+\frac{f(n+1)(1-\beta)^{2}}{c(r+\delta+\gamma)(\delta+\gamma)}\right]+S^{G}\left[\frac{\gamma(1-\beta)}{r+\delta+\gamma}\right] .
\end{aligned}
$$

\section{Appendix 3 : Steady state stocks parameters}

$$
\begin{aligned}
& M_{1}=(r+\delta+\gamma)\left(p b-p_{e}\right)>0, \\
& \mu_{\infty}=-d p /[r+(1-\beta) / \beta]<0,
\end{aligned}
$$




$$
\begin{aligned}
& M_{2}^{s o}=S^{W}(r+\delta+\gamma)+\gamma S^{G}(1-\beta)>0, \\
& M_{2}^{o l}=S^{W}(r+\delta+\gamma)+\gamma_{i} S^{G}(1-\beta)>0, \\
& \lambda_{\infty}^{s o}=\left[-X_{\infty}^{s o}\left(n p f+D \delta S^{G}\right)+\mu_{\infty} \gamma S^{G}\right] /[r+\delta+\gamma], \\
& \lambda_{\infty}^{o l}=\left[-X_{\infty}^{o l} p f+\mu_{\infty} \gamma_{i} S^{G}\right] /[r+\delta+\gamma], \\
& z_{2}^{o l}=(\delta+\gamma)(r+\delta+\gamma)+\frac{n}{p c}(1-\beta)^{2}(p f) .
\end{aligned}
$$

\section{Appendix 4 : Stock paths parameters}

$$
\begin{aligned}
& \mu^{m}(t)=\mu_{\infty}^{m} \\
& \lambda^{m}(t)=\lambda_{\infty}^{m}+\left(\lambda_{0}-\lambda_{\infty}^{m}\right) e^{w_{1}^{m} t}=\lambda_{\infty}^{m}+\left(X_{0}-X_{\infty}^{m}\right) \frac{w_{1 \lambda}^{m}}{w_{1 X}^{m}} e^{w_{1}^{m} t} \\
& D^{s o}=n p f+D \delta S^{G}, D^{o l}=p f \\
& w_{2}=\frac{\beta-1}{\beta}<0 \\
& w_{1}^{m}=\frac{1}{2}\left[r-\sqrt{\left.[r+2(\delta+\gamma)]^{2}+4 n(1-\beta)^{2} D^{m} / p c\right]}<0\right. \\
& w_{1 X}^{m}=\frac{n(1-\beta)\left(\frac{1-\beta}{\beta}+w_{1}^{m}\right)}{n(1-\beta) \gamma_{i} S^{G}+\left(w_{1}^{m}+\delta+\gamma\right) S^{W}} \\
& w_{1 \lambda}^{m}=\frac{\left(w_{1}^{m}-w_{2}\right) p c D^{m}}{S^{W}(1-\beta) D^{m}-\gamma_{i} S^{G} p c\left(r+\delta+\gamma-w_{1}^{m}\right)} \\
& w_{1 Q}^{m}=1, w_{1 \mu}^{m}=0, w_{2 X}^{m}=w_{2 \lambda}^{m}=w_{2 \mu}^{m}=0 \\
& w_{2 Q}^{m}=1
\end{aligned}
$$

\section{Appendix 5 : general model with tax}

$$
\begin{aligned}
& \left(\begin{array}{c}
\dot{X} \\
\dot{\lambda} \\
\dot{Q}_{i} \\
\dot{\mu}
\end{array}\right)=\left(\begin{array}{cccc}
\frac{n(\beta-1)}{p c} \tau_{2}-\delta-\gamma & \frac{n(1-\beta)^{2}}{p c} & 0 & \frac{n S^{W}}{p c}(1-\beta) \\
p f-\frac{\tau_{2}^{2}}{p c} & r+\delta+\gamma+\frac{\tau_{2}(1-\beta)}{p c} & 0 & -\gamma S^{G}+\frac{S^{W}}{p c} \tau_{2} \\
\gamma S^{G}-\frac{S^{W}}{p c} \tau_{2} & \frac{1-\beta}{p c} S^{W} & \frac{\beta-1}{\beta} & \frac{S^{W}}{p c} \\
0 & 0 & 0 & r+\frac{1-\beta}{\beta}
\end{array}\right) \cdot\left(\begin{array}{c}
X \\
\lambda \\
Q_{i} \\
\mu
\end{array}\right)+ \\
& \left(\begin{array}{c}
\frac{n(1-\beta)\left(p b-p_{e}-\tau_{1}\right)}{p c} \\
\tau_{3}+\frac{\tau_{2}\left(p b-p_{e}-\tau_{1}\right)}{p c} \\
\frac{S^{W}\left(p b-p_{e}-\tau_{1}\right)}{p c} \\
d p
\end{array}\right) \\
& z_{2}^{t}=\tau_{2}\left[(\delta+\gamma)(1-\beta) / p c+n(1-\beta)^{2}(r+\delta+\gamma)+(\delta+\gamma)(r+\delta+\gamma)+n(1-\beta)^{2} f / c\right]>0
\end{aligned}
$$




$$
\begin{aligned}
& M_{2}^{t}=M_{2}^{o l}, M_{1}^{t}=\frac{n(1-\beta)}{p c}\left[(r+\delta+\gamma)\left(p b-p_{e}-\tau_{1}\right)-(1-\beta) \tau_{3}\right] \\
& M_{3}^{t}=-\frac{n(1-\beta)}{p c}\left[\tau_{2} \tau_{3}+p f\left(p b-p_{e}-\tau_{1}\right)\right]-(\delta+\gamma)\left[\tau_{3}+\frac{\tau_{2}\left(p b-p_{e}-\tau_{1}\right)}{p c}\right] \\
& M_{4}^{t}=\frac{n(1-\beta)}{p c}\left[S^{W} p f-\gamma S^{G} \tau_{2}\right]+(\delta+\gamma)\left[\frac{S^{W} \tau_{2}}{p c}-\gamma S^{G}\right]
\end{aligned}
$$

